TUBERCULOSIS

\title{
Tuberculosis in contacts need not indicate disease transmission
}

\author{
U R Dahle, S Nordtvedt, B A Winje, T Mannsaaker, E Heldal, P Sandven, H M S Grewal, \\ D A Caugant
}

Thorax 2005;60:136-137. doi: 10.1136/thx.2004.030841

See end of article for authors' affiliations

Correspondence to:

Correspondence to:
Dr U R Dahle, Division of Infectious Disease Control, Norwegian Institute of Public Health, P O Box 4404, Nydalen, $\mathrm{N}-0403$ Oslo, Norway; ulf.dahle@ fhi.no

Received 30 June 2004 Accepted 9 November 2004
Background: Traditional contact investigation is an important tool for controlling tuberculosis. It may also help to indicate drug susceptibility patterns when Mycobacterium tuberculosis cultures are not available. Such investigations often underestimate the degree of transmission found by genotyping, but overestimation may also occur. This report is the result of a routine successive DNA restriction fragment length polymorphism (RFLP) analysis of $M$ tuberculosis isolated in Norway.

Method: Fifteen immigrants belonging to the same community were notified with tuberculosis during February to September 2003. The mycobacterial isolates were analysed by RFLP.

Results: All 15 patients had social contact with each other and 13 belonged to the same church community. A total of 14 cultures were positive for $M$ tuberculosis. Among these isolates, six different genotypes were found. Five patients had not acquired the infection from the putative source.

Conclusions: Reactivation of tuberculosis may occur in contacts during the development of an outbreak. In such situations, traditional contact investigations may overestimate the rate of transmission found by genotyping of $M$ tuberculosis. When cultures are unavailable and presumed drug susceptibility patterns are based on that of contacts, such overestimation may lead to incorrect treatment of a patient. Contact investigations must be combined with genotyping of $M$ tuberculosis to conclude how tuberculosis is transmitted. This is especially important in persons with several risk factors for infection. t has frequently been found that contact tracing underestimates the degree of transmission found by genotyping of Mycobacterium tuberculosis. Such studies have questioned the ability of contact investigations to identify cases in communities. ${ }^{1-5}$ It is, however, also possible that contact tracing sometimes overestimates the rate of transmission. ${ }^{6}$ In this report we present a situation where simultaneous reactivation occurred in contacts during the development of an outbreak. This may be misinterpreted as new transmission.

\section{METHODS}

Fifteen immigrants with social contacts were notified with tuberculosis between 24 February and 1 September 2003. Thirteen belonged to the same religious congregation. All the patients belonged either to the same family, were close friends, or went to the same school. It was therefore believed that an outbreak was ongoing in this community. The mycobacterial isolates were analysed by restriction fragment length polymorphism (RFLP), as described previously, and compared with other isolates in the Norwegian database. ${ }^{7}$ The IS6110 RFLP patterns were compared by visual examination and computer assisted analyses using the BioNumerics Version 1.5 software (Applied Maths, Kortrijk, Belgium). To facilitate the comparison of the fingerprints, normalisation was done using the molecular weight standards run on each gel. Similarity measures were calculated using the Dice coefficient. Cluster analysis was performed using the unweighted pair group average method.

\section{RESULTS}

One patient was culture negative but $M$ tuberculosis was isolated from 14 individuals. All patients (nine female and six male) had pulmonary tuberculosis. Eleven were first generation immigrants who had lived in Norway for between
3 months and 13 years (average 4.5 years). One patient originated from the Philippines and 10 from East Africa. The other four were born in Norway to parents from Ethiopia and five of the subjects were children (aged 1-8 years). The age range of the subjects was $1-43$ years (mean 22.4 years, median 27 years).

One patient lived at a refugee reception centre in Norway and the others lived in private homes at the time of diagnosis. Nine $M$ tuberculosis isolates harboured an identical RFLP pattern (fig 1) which originated from five members of a single family and four friends of this family (including three children). Furthermore, all nine patients attended the same church (a room of $40 \mathrm{~m}^{2}$ ). The other five patients were infected with unique isolates (fig 1). These patients were contacts of the index case or with each other through friendship, school, or church. Two patients who lived at the same address and were notified 2 weeks apart were infected with different strains of $M$ tuberculosis (fig 1). None of these six genotypes had previously been observed in Norway.

\section{DISCUSSION}

This report shows that simultaneous reactivation may occur in contacts during an outbreak in a community of people originating from high incidence countries. The assumed transmission link to the index case is especially important when presuming drug susceptibility patterns in culture negative cases. In this report 15 persons in a community were notified with tuberculosis during a period of 6 months. Based on contact investigations it was reasonable to suspect that an outbreak was ongoing. It was unexpected that six different genotypes of $M$ tuberculosis were found among these persons. The study included 15 patients and generalisations are difficult to make. It was noticed, however, that genotyping of $M$ tuberculosis confirmed the assumed transmission links within the family and for the included children. This 


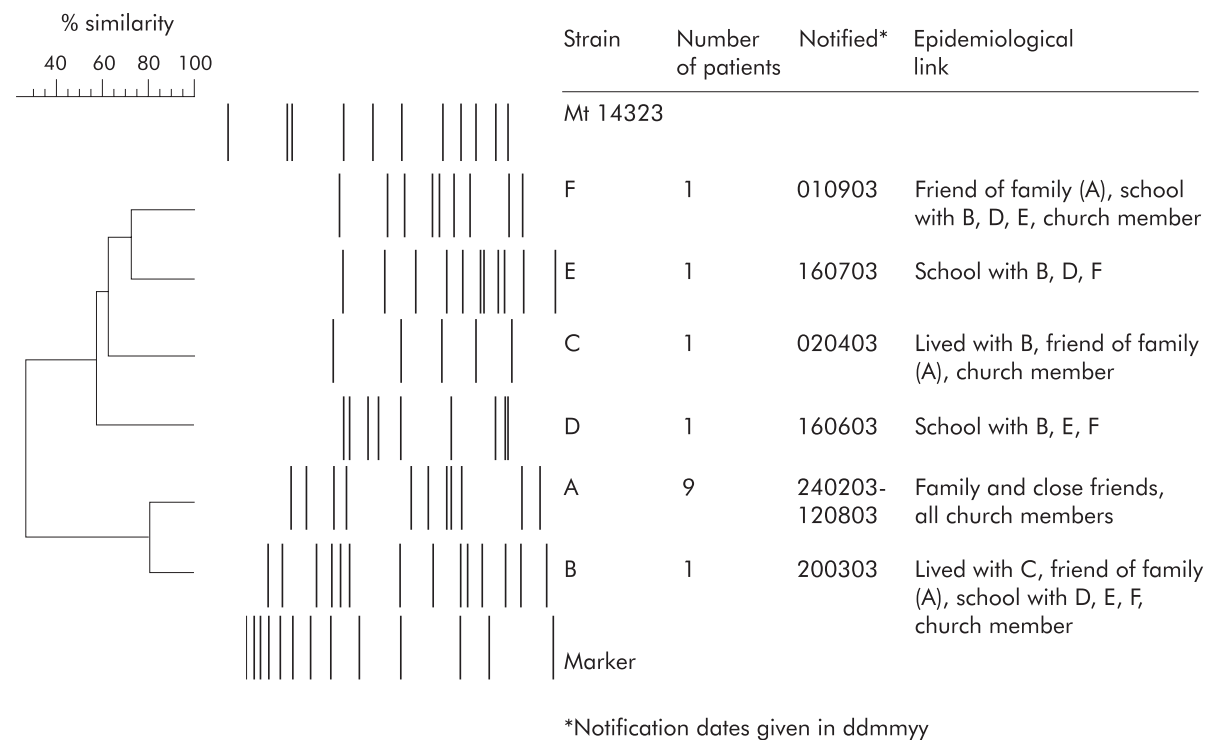

Figure 1 Schematic representation of the similarity of Mycobacterium tuberculosis strains isolated from 14 immigrants in February to September 2003 as well as a reference strain (Mt 14323) and external marker with fragment sizes 12232, 11198, 10180, 9162, 8144, 7126, 6108, 5090, 4072, 3054, 2036, 1635 and 1018 bp. Dates of notification (first and last for each strain) and their epidemiological links are provided.

was not the case for all persons of more distant contact, including a landlord and his tenant.

The observation that five of 13 contacts did not acquire their infection from the putative source does not invalidate the usefulness of contact investigation as a public health intervention. It emphasises, however, that traditional contact tracing needs confirmation by genotyping before transmission patterns of $M$ tuberculosis can be concluded. This is also the case when contact exists, especially between patients with several risk factors for infection.

\section{ACKNOWLEDGEMENTS}

The authors acknowledge the microbiological laboratories in Norway for sending their Mycobacterium tuberculosis isolates to the Norwegian Institute of Public Health and thank Vigdis Dahl, Kjersti Haugum, Bente Forsdahl, Elisabet Rønnild and Ann-Christine Øvrevik for their skillful assistance.

\section{Authors' affiliations}

U R Dahle, B A Winje, T Mannsaaker, E Heldal, P Sandven,

D A Caugant, Division of Infectious Disease Control, Norwegian Institute of Public Health, P O Box 4404, Nydalen, N-0403 Oslo, Norway $S$ Nordtvedt, Department of Thoracic Medicine, Haukeland University Hospital, P O Box 1, N-5021 Bergen, Norway
P Sandven, Institute of Microbiology, Rikshospitalet University Hospital, N-0027 Oslo, Norway

H M S Grewal, Section of Microbiology and Immunology, The Gade Institute, University of Bergen and Haukeland University Hospital, N5021 Bergen, Norway

Competing interests: URD is a scientist working with genotyping of Mycobacterium tuberculosis.

\section{REFERENCES}

1 Daley CL. Tuberculosis contact investigations: please don't fail me now. Am J Respir Crit Care Med 2004; 169:779-81.

2 Ruddy MC, Davies AP, Yates MD, et al. Outbreak of isoniazid resistant tuberculosis in north London. Thorax 2004;59:279-85.

3 Small PM, Hopewell PC, Singh SP, et al. The contemporary urban epidemiology of tuberculosis: a population-based study using conventional and molecular methods. N Engl J Med 1994;330:1703-9.

4 van Deutekom $\mathrm{H}$, Hoijng SP, de Haas PEW, et al. Clustered tuberculosis cases: do they represent recent transmission and can they be detected earlier? Am J Respir Crit Care Med 2004; 169:806-10.

5 Heldal E, Dahle UR, Sandven P, et al. Risk factors for recent transmission of Mycobacterium tuberculosis in Norway. Eur Respir J 2003:21:637-42.

6 Behr MA, Hopewell PC, Paz EA, et al. Predictive value of contact investigation for identifying recent transmission of Mycobacterium tuberculosis. Am J Respir Crit Care Med 1998; 158:465-9.

7 Dahle UR, Sandven P, Heldal E, et al. Continued low rates of transmission of Mycobacterium tuberculosis in Norway. J Clin Microbiol 2003;41:2968-73. 